\title{
COMPOSITION MEASUREMENT ABOVE 1 EeV WITH A LARGE ARRAY NEAR THE HIGH RESOLUTION FLY'S EYE
}

\author{
K.D. Green \\ University of Michigan
}

The purpose of this workshop is to design a detector which will shed light on the origin of cosmic rays at the highest known energies. The emphasis has been on detecting cosmic rays with energies above $10 \mathrm{EeV}$ with the specific goals of testing the Greisen-Zatsepin hypothesis, searching for anisotropy, and inferring composition of the primaries. These are noble goals, but there is value in concentrating on understanding these properties above $E=1 E e V$.

The previous experimental designs (mainly ground arrays) have focused principally on anisotropy, then spectrum, and most poorly, composition. There are practical reasons for this. For one, anisotropy is generally easier to measure than composition. However, barring the detection of point sources, composition needs to be known if propagation and the acceleration mechanism are to be understocd. Further, composition information permits highly discriminating anisotropy and spectral change analyses.

It is my opinion that focusing on composition is a prudent next step. The proposed detector consists of a ground array in the vicinity of the High Resolution (HiRes) Fly's Eye. Building and operating an array is not only useful in refining experimental techniques for the grander proposal of $10^{4} \mathrm{~km}^{2} \mathrm{sr}$, but in conjunction with the HiRes Fly's Eye, the combination will clarify certain outstanding questions regarding EAS phenomenology and cosmic ray acceleration processes. This proposal necessarily fails to satisfy one of the original requirements of the workshop design criteria; namely a time averaged $10^{4} \mathrm{~km}^{2} \mathrm{sr}$ collection area above $100 \mathrm{EeV}$. Arguments can be made that an interesting energy region begins near $1 \mathrm{EeV}$. It may also be possible that a large array similar to those proposed at the workshop[1],[2] situated near the HiRes Fly's Eye will satisfy the necessary design requirements for a useful composition measurement.

This proposal is not new. It has been suggested many times in the past. [3],[4]

The paper is divided into three sections. First, empirical and presumed cosmic ray properties are discussed in an attempt to justify both composition and $1 E e V$ as the areas of focus. Second, techniques to measure composition are discussed. Third, estimates of the design requirements and capabilities of the current and proposed detectors are discussed.

\section{Why Composition Above $1 \mathrm{EeV}$ is Interesting}

A convincing argument can be made that en energy region of interest begins just below the "knee" energy where a spectral change is known to occur. Since this conference concenirates on the highest known energy particles, the discussion is tailored to these highest energies in which case $1 \mathrm{EeV}$ appears to be a natural threshold energy. There are also practical reasons for this choice. The optimal detector design 
for the energy range above $0.1 \mathrm{EeV}$ is necessarily different than 0.1PeV. Also, HiRes/CASA/MIA will exist as a prototype of the proposed design and will operate in the energy region of $0.05-0.5 \mathrm{EeV}$. However, practicalities aren't the only arguments.

The Fly's Eye $\left\langle X_{\max }\right\rangle$ and $\sigma_{X_{\max }}$ measurements above $0.1 \mathrm{EeV}$ [5] suggest the cosmic ray composition is 'mixed' up to $10 \mathrm{EeV}$. The data are consistent with a simplistic 2-component model of $50 \% \mathrm{Fe}, 50 \% \mathrm{H}$. The Akeno $\left\langle N_{\mu}\right\rangle \mathrm{pa}-$ rameterization[6] and the Haverah Park measurement of shower arrival time fluctuations[7] are also consistent with a large fraction of heavy nuclei. This key point of mixed composition is an important feature in the argument for the proposed design. It is difficult theoretically to create a model which keeps the composition constant in the range of $1-100 \mathrm{EeV}$.

The current technologies are perhaps resolving spectral structure in the form of a "dip" and "ankle" in the $1-10 E e V$ region. As recently reviewed by Lloyd-Evans[8], there are no statistically compelling broad band anisotropies above $0.1 \mathrm{EeV}$, but there is some evidence for a galactic enhancement from $5 \mathrm{EeV}$ to $15 \mathrm{EeV}$ with a statistical significance near the $1 \%$ level. The most compelling aspect of the anisotropy measurements are the fact that the anisostropy begins near the place where the "dip" begins. The issue of the statistical significance of these results should be resolved with data from AGASA and EAS-1000 in the next few years. Assume for the moment that the observed spectral structure is still present with the larger dataset, but the anisotropy isn't. Then it is not clear if some unkown systematic cause of the observed spectral structure would be found by the present designs.

Given a mixed composition, first consider a galactic origin of cosmic rays of energies up to and perhaps beyond $1 E e V$. This approach is consistent with the general picture of Axford.[9] A galactic enhancement should become apparent if the cosmic rays originate along the disk.
Assuming an enhancement is confirmed, then there should be a correlation between an increasing anisotropy and a reduction in $(Z\rangle$. The composition should be lighter for showers coming from the galactic plane, and again, assuming a predominately galactic origin, the composition should be heavier for showers arriving off-plane. In this case, a composition measurement in this energy region would be the logical next step to eliminate any systematic detector bias which might produce the results. This argument can be turned around as well. Selecting events by $\langle Z\rangle$ bins could reveal expected galactic anisotropies. A preliminary at lateral slope information from the world's largest arrays has recently been published. This attempt has been criticized by experimenters who believe the lateral slope sensitivity is inaccessable with the present detectors.[8] In principle, searching for anisotropy in different $(Z)$ regions is a good idea, provided the composition can be reliably binned.

Next consider an increasing extragalactic component in the energy region above the "knee." Photodisintegration may become an important process near $1-5 \mathrm{EeV}$ for nuclei.[11] This process has an effect on the propagated composition. Photodisintegration will begin to remove the heavier composition, principally leaving only a light composition above $10 \mathrm{EeV}$. Thus, the Greisen-Zatsepin hypothesis is testable at a more accessable energy region provided a mixed cosmic ray source composition continues through $10 \mathrm{EeV}$ and the propagation lengths are between $10-1000 M p c$. This theory can be systematically tested provided good composition information is known above $1 \mathrm{EeV}$.

In these cases considered, a change in composition above $1 \mathrm{EeV}$ and before $100 \mathrm{EeV}$ occurs. Further, the microwave background cutoff can be tested at an energy region experimentally more accessible provided there is a "mixed" source composition.

Finally, assume an origin which is extra- 
galactic, but "local" ( $<100 \mathrm{Mpc}$ ). In this case significant fractions of all species will survive. The composition can be inferred from anisotropy measurements above $100 \mathrm{EeV}$ provided such anisotropies are detectable. However, the particle charge and the magnetic fields encountered during propagation may not be separable, and thus an ambiguity can arise about the exact acceleration process. If the acceleration mechanism is to be understood, the composition must be known. Further, given the composition distribution and an anisotropy, the propagation pathlengths are experimentally accessible. I wish to pose questions directed to designers of large arrays: Can anisotropy and energy convey enough information by themselves to reveal the acceleration mechanism? Can one distinguish between a cutoff of 'universal' protons from a principally heavy composition produced 'locally' and whose source spectrum cuts off near $100 \mathrm{EeV}$ as some theories suggest[9] without knowing the primary composition?

\section{Techniques to Measure Composition above}

\subsection{EeV}

All air shower techniques for measuring composition are indirect. Most attempts try to measure quantities sensitive to the initial interaction depth, $X_{0}$, because the protons interact deeper than do heavier elements. At present, the Fly's Eye $X_{\max }$ measurement[5] is preferred, because it measures $X_{\max }$ directly, and $X_{\max }$ is currently the measured parameter which is most also sensitive to $X_{0}$ and, hence, to the atomic number $A$. In principle the lateral distribution slopes are sensitive to $X_{\max }$.[12] Herein lies one justification to construct an array near the the HiRes Fly's Eye; namely, an experimental test of the sensitivity of ground arrays to infer $\langle A\rangle$ by comparing with the HiRes $X_{\max }$.

Another approach to determine composition uses the muon component of the EAS as a probe of the hadronic interactions. While the high en- ergy muons $(E>1 \mathrm{TeV})$ probe the earlier interactions, here only the low energy muons $(E>$ $1 \mathrm{GeV}$ ) are discussed for practical reasons. According to Elbert and Gaisser,[13] the total number of muons at a depth of $1000 \mathrm{~g}-\mathrm{cm}^{-2}\left(30^{\circ}\right.$ at Dugway, Utah) for a primary with atomic number $A$ and energy $E_{p}$ can be expressed as

$$
N_{\mu}=.048 A\left(\frac{E_{p}(G e V)}{A}\right)^{.86} \text {. }
$$

This formula is based on simulations of EAS in the energy region $10^{12}-10^{18} \mathrm{eV}$. The model used to calculate this expression assumes a superposition model with scaling and a $\log ^{2} s$ cross section.

Not only is the total number of muons composition sensitive, but so is the shape of the lateral distribution.[3] Separation of heavy and light primaries is possible at large core distances, because the muons produced in a heavy nucleon shower are generated at higher altitudes than those produced from a lighter nucleon shower and thereby will fall at large core distances. This feature will not be explicitly exploited in this paper, because the difference in lateral distribution is in part due to the difference in $\left\langle X_{0}\right\rangle$.

Both the $X_{\max }$ and $N_{\mu}$ techniques require monte carlo guidance. Work in the eñergy range above $0.1 \mathrm{EeV}$ has understandably concentrated on the $X_{\max }$ technique. Recently, nucleus-nucleus interactions have been modelled using a wounded nucleon picture as opposed to simple superposition for heavier nuclei.[14] The results give $\left\langle X_{\max }\right\rangle$ values consistent with superposition, but $\sigma_{X_{\max }}$ is larger than what superposition predicts. Table 1 lists $N_{\mu}$ and $X_{\max }$ for showers of different compositions and energies. Bear in mind the models used to calculate $X_{\max }$ and $N_{\mu}$ are slightly different, and $N_{\mu}$ is extrapolated above $1 \mathrm{EeV}$. How fluctuations in $N_{\mu}$ behave still requires detailed monte carlo analysis. For now, assume

$$
\frac{\sigma_{N_{\mu}}}{N_{\mu}}=\frac{0.20}{\sqrt{4}}
$$

This general formula is consistent with 
the model of hadronic interaction used by the Yakutsk group[18] and is in qualitative agreement with extrapolations from lower energies of the superposition model.[19]

To determine composition it will be important to understand details of the nuclear interactions. A variety of interaction models have been proposed and their predictions of $X_{\max }$ and $\sigma_{X_{\max }}$ have been calculated for proton initiated showers.[15] Ignorance of the actual interaction processes limits any conclusion regarding composition. For example, if the actual muon multiplicity is higher than assumed, showers will interact earlier, so a proton dominant composition could mimic showers from heavier primaries. The HiRes will have some constraining ability, because $\sigma_{X_{\max }}$ is also sensitive to multiplicity. However, muons will also help constrain the multiplicity provided this hypothesized multiplicity rise is not composition sensitive. Thus it is conjectured that muon information can help clarify the situation, but the sensitivity of a muon constraint to different interaction models must still be quantitatively determined.

\section{Design Parameters Needed for Composition Measurement}

This section discusses general design criteria. While it gives only a few specific array requirements, the discussion is nonetheless useful for it points out important difficulties which must be overcome.

A detector's $X_{\max }$ resolution should be less than the predicted shower fluctuation width in $X_{\max }$. Fe initiated showers fluctuate by $\sigma_{X_{\max }} \approx$ $35 \mathrm{~g} \mathrm{~cm}^{-2}$. The HiRes $X_{\max }$ resolution is expected to be $\delta X_{\max }= \pm 30 \mathrm{~g} \mathrm{~cm}^{-2}$ for showers detected in the energy range $0.1-100 \mathrm{EeV} .[16]$ The HiRes is presently the only detector which can measure $X_{\max }$ to this desired accuracy in a model independent way which is a strong reason for using it. However, we must presently assume that $\delta X_{\max }$ and $\delta E / E$ will not degrade
TABLE 1. $\left\langle X_{\max }\right\rangle$ and $\left\langle N_{\mu}\right\rangle$ as functions of energy and $A$ from an energy spectrum with an index of -3.0. The $\sigma$ 's represent the intrinsic or "natural" widths of $X_{\max }$ and $N_{\mu}$ for each energy bin. No detector uncertainty has been added. $X_{\max }$ and $\sigma_{X_{\max }}$ are based on "wounded nucleon" simulations, while $N_{\mu}$ and $\sigma_{N_{\mu}}$ are consistent with simple superposition models.

\begin{tabular}{|c|c|c|c|}
\hline $\begin{array}{c}\mathrm{E} \\
(\mathrm{EeV})\end{array}$ & $A$ & $\begin{array}{c}X_{\max } \pm \sigma_{X_{\operatorname{mex}}} \\
\left(g \mathrm{~cm}^{-2}\right)\end{array}$ & $\begin{array}{c}N_{\mu} \pm \sigma_{N_{\mu}}\left(10^{6}\right) \\
(E>1 G e V)\end{array}$ \\
\hline $0.1-0.3$ & $\mathrm{H}$ & $721 \pm 63$ & $.52 \pm .18$ \\
& $\mathrm{C}$ & $658 \pm 42$ & $.72 \pm .19$ \\
& $\mathrm{Fe}$ & $617 \pm 38$ & $.90 \pm .24$ \\
\hline $1.0-3.0$ & $\mathrm{H}$ & $772 \pm 57$ & $3.8 \pm 1.3$ \\
& $\mathrm{C}$ & $720 \pm 40$ & $5.2 \pm 1.4$ \\
& $\mathrm{Fe}$ & $678 \pm 35$ & $6.5 \pm 1.7$ \\
\hline $3.0-10$. & $\mathrm{H}$ & $801 \pm 57$ & $9.8 \pm 3.5$ \\
& $\mathrm{C}$ & $746 \pm 39$ & $14.0 \pm 4.2$ \\
& $\mathrm{Fe}$ & $707 \pm 34$ & $17.4 \pm 4.9$ \\
\hline $10 .-30$. & $\mathrm{H}$ & $834 \pm 55$ & $27 . \pm 9$. \\
& $\mathrm{C}$ & $775 \pm 41$ & $38 . \pm 10$. \\
& $\mathrm{Fe}$ & $736 \pm 34$ & $47 . \pm 13$. \\
\hline
\end{tabular}

significantly by substituting an array for a second HiRes site.

The HiRes estimated energy resolution is $\delta E=20 \%$ at $1 E e V$ which is also obtained through means which are virtually model independent. Slight model dependence is required to estimate the amount of undetected energy $(\approx 10 \%)$. A muon measurement will reduce the uncertainty of this correction.

A tolerable experimental uncertainty in $N_{\mu}$ partially depends on the natural $N_{\mu}$ fluctuations which are presently under study.[17] For this discussion, assume the number of muons in showers fluctuate as quoted above. Because they can be corrected for to some extent, assume there are no intrinsic variations with core distance, zenith angle, or $X_{0}$ in the muon lateral distribution which would cause an uncertainty larger than the above formula gives.

Experimental uncertainty in measuring $N_{\mu}$ is caused by (1) the core and angular resolution, 
and (2) the measurable dynamic range of $\rho_{\mu}$, (3) intrinsice shower fluctuations, and (4) experimental sampling. The core resolution and deciector spacing are discussed here, because the sampling determines a significant part of the detector cost.

For full trigger efficiency $1 E \& V$, the maximum the detector spacing can be is $0.15-1.0 \mathrm{~km}$ over the area where the HiRes also triggers.[1] The minimum the detector spacing needs to be depends on the acceptable core error and shower sampling.

The core uncertainty's contribution to the muon density resolution, $\delta \rho_{\mu}$ (and hence $\delta N_{\mu}$ ), can be estimated from the Fly's Eye/MIA muon data[20] which are consistent with the Yakutsk measured muon lateral distribution parameterization[21] scaled to Dugway depths. For $1 \mathrm{EeV}$ showers at a zenith angle of $30^{\circ}$, a gaussian distributed $50 \mathrm{~m}$ core error translates into a single density measurement uncertainty of $25 \%$, $15 \%, 10 \%$ at $R_{\text {core }}=400 \mathrm{~m}, 800 \mathrm{~m}, 1200 \mathrm{~m}$, respectively. Multiple measurements will reduce the detector error only if measurements are averaged on more than one side of the shower. The proposed design allows for events lying outside the array boundary to be used provided the core error is small enough. The design goal requires $\delta R_{\text {core }}<50 m$ for these events. How this may be possible is discussed below. For showers falling inside the array boundary, the core uncertainty from a simple weighted average core estimate is $13 \%$ of the detector spacing.[1] A $0.75 \mathrm{~km}$ spacing thus gives a $100 \mathrm{~m}$ core error which is probably sufficient because of the multiple sampling ( $\geq 10$ alerted stations above $1 E e V$ ). Also, through a combined HiRes-Array analysis technique discussed below, the core error will most likely be smaller. Thus, the core error contribuiion will not require the counter spacing to be closer. The remaining question on the tolerability of a $0.75 \mathrm{~km}$ spacing is if the sampling is adequate for composition analysis. This rests critically on how well the lateral distribution must

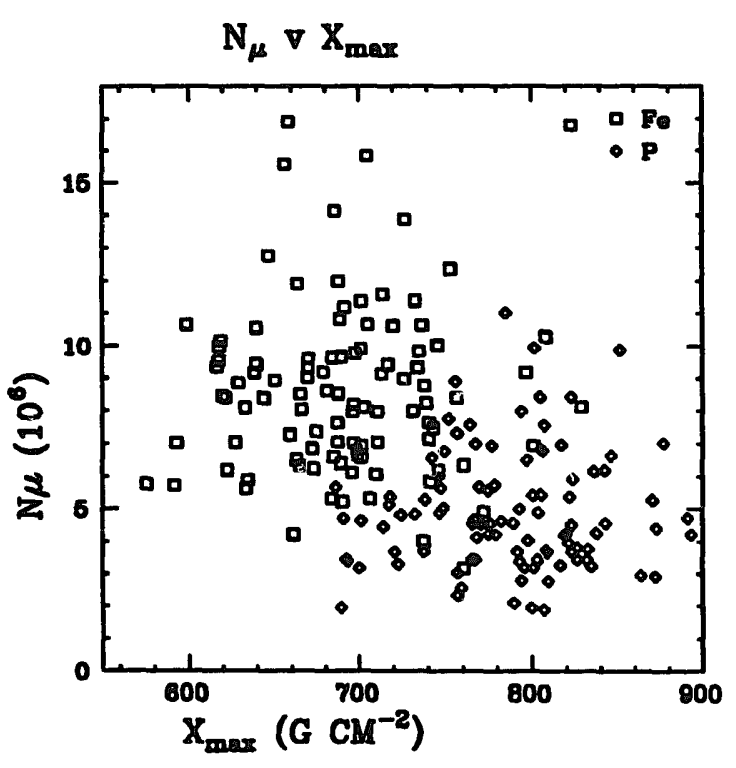

Fig. 1. The expected $X_{\max }$ v. $N_{\mu}$ distribution for a realistic detector design. Relevant design parameters are listed in the text.

be determined event by event.

For purposes of discussion, assume all experimental uncertainties give $\delta N_{\mu} / N_{\mu}=.20$.

Figure 1 shows the expected $X_{\max }$ and $N_{\mu}$ correlation for an $E^{-3}$ input spectrum with $50 \%$ Fe and $50 \% \mathrm{H}$ assuming the stated natural variations and experimental uncertainties. $X_{\max }$ and $N_{\mu}$ have been deconvoluted to $E=2.0 \mathrm{EeV}$. In other words, for each event, $X_{\max }$ and $N_{\mu}$ are corrected to the values of a similar shower at $2.0 \mathrm{EeV}$ based on an estimated energy whose uncertainty follows a gaussian distribution with a width of $20 \%$. The trigger efficiency is assumed to be $100 \%$ above $1 \mathrm{EeV}$ and $0 \%$ below. Distinct separation of the two components is possible using both parameters. This can be considered the minimally acceptable experimental design goal.

Figure 2 shows an optimal design case where the natural fluctuations are as assumed above, but the experimental errors are $\delta X_{\max }=5 \mathrm{gcm}^{-2}, \delta E / E=.05, \delta N_{\mu} / N_{\mu}=.05$. Carbon has been added to the plot. While 
it is acknowledged the muon fluctuations are most likely underestimated, it is worth pointing out that the three components are distinctly resolvable by $N_{\mu}$ alone. The parameters of interest for better resolution of $\langle A\rangle$ are currently $\delta E / E$ and $\delta N_{\mu} / N_{\mu}$ and not $X_{\max }$ (provided the design criterium of $\delta X_{\max }=30 \mathrm{~g} \mathrm{~cm}^{-2}$ is met). For composition studies, this shows the value of steering the near future direction of improvements towards better energy and muon resolution. Better energy resolution is perhaps justification enough for a surface electron array along with a muon detector.

Figure 2 also reveals the ultimate limitation of the $X_{\max }$ technique. To move beyond this limitation, detailed shower fluctuations must be understood. It is difficult to see how this will be done with any ground array by itself. Air fluorescence may have the capability by looking at the entire longitudinal distribution; particularly fluctuations in the early part of the shower.[22] In practice, muon information will be useful in constraining shower interaction models.

The HiRes prototype and CASA/MIA[23] can be considered as a prototype for testing the ideas presented in this paper. The coincidence aperture has a threshold at $0.05 \mathrm{EeV}$ with an aperture $\approx 5 \mathrm{~km}^{2} \mathrm{sr}$ climbing to $\approx 50 \mathrm{~km}^{2} \mathrm{sr}$ at $0.5 \mathrm{EeV}$. However, the aperture for wellreconstructed events must necessarily be smaller.

For HiRes/CASA/MIA detected showers whose cores are "sufficiently contained" inside CASA, the event geometry can be determined by CASA alone $\left(\delta R_{\text {core }}<10 \mathrm{~m}, \delta \theta_{z}<.5^{\circ}\right.$ for these large showers). Unfortunately, events must be inside the $0.25 \mathrm{~km}^{2}$ array boundary for this resolution. MIA will begin to saturate within $500 \mathrm{~m}$ of the core near the energy region of $0.3-0.5 \mathrm{EeV}$, but the data rate above this energy for this limited aperture is $<10 /$ year. The expected rate for contained showers is $\sim 1700 /$ year.

For showers falling outside CASA, it is unlikely HiRes differential timing information by

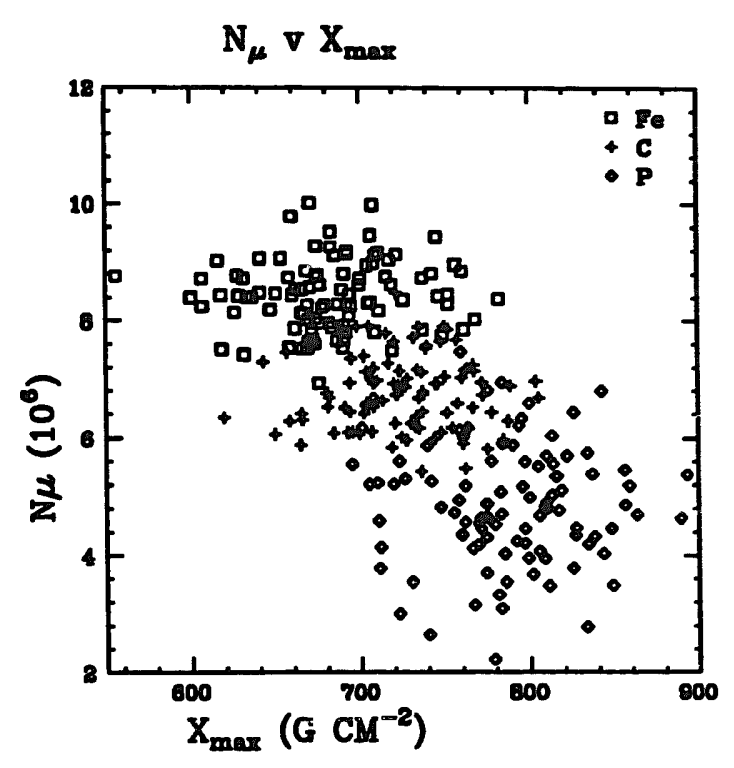

Fig. 2. The expected $X_{\max }$ v. $N_{\mu}$ distribution for an optimized design. Relevant design parameters are listed in the text.

itself can reliably reconstruct the core location.[24] It is possible a combined reconstruction technique could provide the required accuracy. In particular, the geometry can be determined by the overlapping constraints provided by the HiRes shower plane $\left(0.2^{\circ}\right.$ resolution[25]), the ground array direction ( $<2^{\circ}$ resolution), and the HiRes differential timing fit.[26] The core resolution would approach the design requirement of $\delta R_{\text {core }}<50 \mathrm{~m}$. It is possible this constrained differential timing/direction method could be used for the proposed HiRes-Array to provide the required core accuracy. Tests with HiRes/CASA/MIA are planned to determine the potential usefulness of this technique.

Another potentially useful method to determine the shower geometry is to use synchronized timing information from the HiRes and the array. The idea is to use the time the shower front passes through the ground array in the HiRes timing fit. The method requires the HiRes and array clocks be synckronized to high accu- 
racy. A crude estimate on the core resolution is the resolution with which one can synchronize the two experiments. Thus, 100nsec will give $\delta R_{\text {core }} \approx 30 \mathrm{~m}$ at best. There is clearly a premium in minimizing this time. In March, 1990, the MIA electronics were modified to record the arrival time of the Fly's Eye trigger. On the Fly's Eyes' side, the time a trigger pulse is sent from the Fly's Eye to MIA is recorded. Determining the utility of this new fitting technique is a work in progress. A crude value of the expected angular resolution has already been estimated to be $<1^{\circ}$.[27]

\section{Conclusions}

Since composition is crucial is understanding acceleration mechanisms and propagation, efforts should focus on improving the ability to distinguish the different species. Other advantages of building an array near the HiRes include a valuable energy cross-calibration and further consistency checks between the two techniques. Dual separation of light and heavy nuclei above $1 E e V$ is experimentally possible with a large muon array near the HiRes Fly's Eye. Further improvements in nucleon resolution requires "going beyond $X_{\max }$ " to other techniques.

\section{References}

[1] Cronin, Design Concept for a $5000 \mathrm{~km}^{2}$ Air Shower Array, This conference.

[2] Green, et al., Design Parameters for a $10^{20}$ eV Array, This conference.

[3] Elbert, Possible Experiments Using Fly's-Eye-Type Detectors with Other Detector Types, UU-HEP $81 / 1$.
[4] Yodh, Proc. Air Shower Workshop, (Utah) 112, (10 Jan 1383).

[5] Cassiday, et al., Astrophys. J, 356, 669 (1990).

[6] Nagano, et al., J. Phys. G10, 1295 (1984).

[7] Walker and Watson, J. Phys. G8, (1982).

[8] Lloyd-Evans, Proc. 22 ${ }^{\text {nd }}$ ICRC 5, p215 (Dublin) (1991).

[9] Axford, Astrophysical Aspects of the Most Energetic Cosmic Rays, ed: Nagano, Takahama, (World Scientific), 406 (1991).

[10] Chi et al., J. Phys. G18, 539 (1992).

[11] Puget, Stecker, Bredekamp, 205, Astrophys. J., 638, (1976).

[12] Patterson and Hillas, J. Phys. G9, 1433 (1983).

[13] Elbert and Gaisser, Proc $16^{\text {th }}$ ICRC, 8, 42 (1979) (Kyoto).

[14] Engel, et al., Proc 22 ${ }^{\text {nd }}$ ICRC, 4, 1 (1991) (Dublin).

[15] Gaisser, et al., Proc 22 ${ }^{\text {nd }}$ ICRC, 4, 413 (1991) (Dublin).

[16] Cassiday, et al., U. of Utah, HiRes Proposal (1991).

[17] Dave Kieda, U. of Utah, private communication (1992).

[18] Khristiansen, et al., Astrophysical Aspects of the Most Energetic Cosmic Rays, ed: Nagano, Takahama, (World Scientific), 354 (1991).

[19] Jim Matthews, U. of Michigan, private communication (1992).

[20] Cassiday, et al., Proc 21 st ICRC, (Adelaide), 9, 118, (1990).

[21] Dyakonov et al., Proc. 20 ${ }^{\text {th }}$ ICRC, (Moscow), 5, 486, (1987).

[22] Gaisser, Proc. Air Shower Workshop, (Utah) 57, (15 May 1979).

[23] Fick, et al., Porc. 22 ${ }^{\text {rd }}$ ICRC, (Dublin), 2, 728, (1991).

[24] H.Y. Dai, U. of Utah, private communication (1992).

[25] E.C. Loh, A Hybrid (HiRes-Surface Array) Detector, This conference.

[26] Green, AIP Conference Proc. 220184 (1990).

[27] P. Sommers, U of Utah, UU-HiRes, March 11, 1992. This conference. 